\title{
Study of electric field enhanced emission rates of an electron trap in $n$-type GaN grown by hydride vapor phase epitaxy
}

\author{
H. Ashraf, ${ }^{1}$ M. Imran $\operatorname{Arshad}^{2}$ S. M. Faraz, ${ }^{3,4}$ Q. Wahab, ${ }^{3}$ P. R. Hageman, ${ }^{1, a)}$ and \\ M. Asghar ${ }^{2}$ \\ ${ }^{1}$ Applied Material Science, Institute for Molecules and Materials (IMM), Radboud University Nijmegen, \\ Heyendaalsweg 135, 6525 AJ Nijmegen, The Netherlands \\ ${ }^{2}$ Department of Physics, The Islamia University of Bahawalpur, Bahawalpur 63100, Pakistan \\ ${ }^{3}$ Depatment of Physics, Chemistry and Biology (IFM), Linköping University, 58183 Linköping, Sweden \\ ${ }^{4}$ Electronic Engineering Department, NED Engineering University, Karachi 75270, Pakistan
}

(Received 8 May 2010; accepted 13 September 2010; published online 18 November 2010)

\begin{abstract}
Electric field-enhanced emission of electrons from a deep level defect in GaN grown by hydride vapor phase epitaxy has been studied. Using the field dependent mode of conventional deep level transient spectroscopy (DLTS), several frequency scans were performed keeping applied electric field $(12.8-31.4 \mathrm{MV} / \mathrm{m})$ and sample temperature $(300-360 \mathrm{~K})$ constant. Arrhenius plots of the resultant data yielded an activation energy of the electron trap $E$ ranging from $E_{c}$ $-0.48 \pm 0.02 \mathrm{eV}$ to $E_{c}-0.35 \pm 0.02 \mathrm{eV}$, respectively. The extrapolation of the as-measured field dependent data (activation energy) revealed the zero-field emission energy (pure thermal activation energy) of the trap to be $0.55 \pm 0.02 \mathrm{eV}$. Various theoretical models were applied to justify the field-enhanced emission of the carriers from the trap. Eventually it was found that the Poole-Frenkel model associated with a square well potential of radius $r=4.8 \mathrm{~nm}$ was consistent with the experimental data, and, as a result, the trap is attributed to a charged impurity. Earlier, qualitative measurements like current-voltage $(I-V)$ and capacitance-voltage $(C-V)$ measurements were performed, and screening parameters of the device were extracted to ascertain the reliability of DLTS data. (C) 2010 American Institute of Physics. [doi:10.1063/1.3499669]
\end{abstract}

\section{INTRODUCTION}

In the present electronics industry, wide band gap semiconductors such as $\mathrm{GaN}, \mathrm{ZnO}, \mathrm{SiC}$, and related III-V nitride compounds are commonly used for electronic and optoelectronic devices. $\mathrm{GaN}$ is an attractive material with a band gap $3.39 \mathrm{eV}$, a strong breakdown electric field strength 2 $\times 10^{6} \mathrm{~V} / \mathrm{cm}$, and a high saturation carrier velocity 2.7 $\times 10^{7} \mathrm{~cm} / \mathrm{s}$. These properties make it useful in high frequency and high power electronic and optoelectronic devices. $^{1-3}$ Some typical applications are blue light-emitting diodes, laser diodes, violet/green electroluminescence, and high electron mobility transistors with a power density of above $32 \mathrm{~W} / \mathrm{mm}$ together with power efficiency of $54 \%$ at 4 $\mathrm{GHz}^{4-6}$ The material quality of $\mathrm{GaN}$ in terms of carrier mobility, $p$-type doping and doping uniformity has been improved greatly in the recent years. Device processing has been developed together with good Ohmic and Schottky contacts, but, nevertheless, improvement in the device performance is not an easy task, because surface states and deep level defects have detrimental effects, acting either as carrier traps and/or generation-recombination centers. For example, intrinsic point defects and their clusters can act as nonradiative carrier recombination sites and reduce light-emission efficiency. ${ }^{7}$ Different research groups, using deep level transient spectroscopy, ${ }^{8-21}$ have reported a number of deep level defects having activation energy ranging from 0.10 to 0.94 $\mathrm{eV}$ in $\mathrm{GaN}$ grown by different techniques such as metal-

${ }^{\text {a)} E l e c t r o n i c ~ m a i l: ~ p . h a g e m a n @ ~ @ ~ s c i e n c e . r u . n l . ~}$ organic chemical-vapor deposition, molecular beam epitaxy, and hydride vapor phase epitaxy (HVPE). Among these defects, an electron level, referred as level $E$ having activation energy in the range of $0.53-0.61 \mathrm{eV}$ is still under investigation because its nature is yet to be established in view of its electric field-enhanced emission properties. According to Soh et al. ${ }^{14}$ level $E$ possesses logarithmic capture behavior, $\mathrm{Wu}$ et al. reported it to exhibit metastable behavior if annealed under applied field, ${ }^{15}$ and Asghar et al. ${ }^{16}$ reported the field dependent transformation of this level from an electrically active state into an inactive state. Recently, feeling the sensitivity of the issue, Pernot and Muret, ${ }^{21}$ preferred spacecharge depth modulation, ${ }^{22}$ based on the well established Arrhenius principle ${ }^{23}$ to report the temperature dependence $(250-330 \mathrm{~K})$ of free energy $E_{a}$ and capture cross section $\sigma$ of the trap $E$ ( $0.6 \mathrm{eV}$ below the conduction band minimum) in $n$-GaN using DLTS. As a result, they proposed the level to be a neutral charge center. Yet in the most recent past, Polyakov et $a l .{ }^{24}$ observed its (trap E:0.6 eV) shifting to the vicinity of the shallower $(0.25 \mathrm{eV})$ trap in two similar samples of GaN. So the nature of this defect is still not unambiguously established and deeper investigations are necessary and worthwhile.

Under this scenario, we report an investigation leading to ascertain a quantitative enhancement of the emission rate (activation energy) of this level as a function of electric field at constant temperature using field-dependence mode of the DLTS setup. The acquired data have been tested with the available theoretical models: Poole-Frenkel and phononassisted tunneling associated with a Columbic potential, 


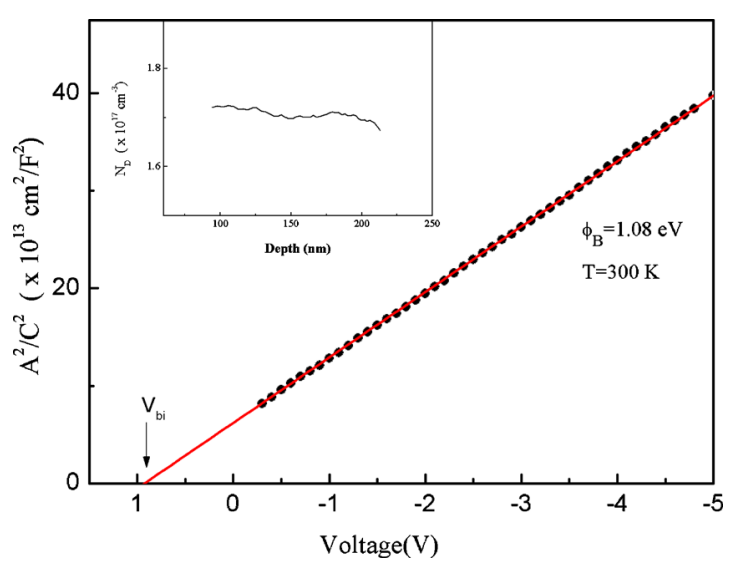

FIG. 1. (Color online) Typical $C$ - $V$ measurements performed at $75 \mathrm{kHz}$ to evidence Schottky behavior of the $\mathrm{Au} / \mathrm{GaN}$ device from $A^{2} / C^{2}-V$ linear relationship. Inset witnesses homogeneous spatial distribution of free carrier.

square well potential, and polarization potential, etc. Consequently, the level has been found to be a charged impurity. Experimental details, results, discussion and conclusions are presented in Secs. II-IV, respectively.

\section{EXPERIMENTAL DETAIL}

Samples investigated in the current study were grown in a HVPE reactor, which had a horizontal configuration. ${ }^{25}$ The $\mathrm{Ga}$ precursor employed for the growth process was $\mathrm{GaCl}$ formed by flowing at $1000{ }^{\circ} \mathrm{C}$ over a gallium (Ga) melt. These $\mathrm{GaCl}$ species reacted with a flow of gaseous ammonia $\left(\mathrm{NH}_{3}\right)$ above the sapphire substrate to form gallium nitride $(\mathrm{GaN})$ species. GaN samples with thicknesses of 550 to $600 \mu \mathrm{m}$ were grown in two successive runs. During cooling down of the samples after the second growth run, the GaN layers spontaneously separated from the sapphire substrate in large pieces due to high compressive strain. ${ }^{26}$ In order to perform electrical characterization of the layers, Schottky and Ohmic contacts were made by evaporating metals on the Ga polar (0001) surface. First the Ohmic contacts were made by evaporating a $100 \mathrm{~nm}$ thick aluminum (Al) layer followed by the deposition of a $20 \mathrm{~nm}$ thick titanium (Ti) layer (which saves Al from oxidation) and annealing at $300{ }^{\circ} \mathrm{C}$ for $3 \mathrm{~min}$. Secondly, the circular Schottky contacts with a diameter of 1 $\mathrm{mm}$ were fabricated by sequentially evaporating palladium $(\mathrm{Pd})$, titanium $(\mathrm{Ti})$, and gold $(\mathrm{Au})$ layers with thicknesses of 40, 20, and $160 \mathrm{~nm}$, respectively, through a shadow mask. Here Pd and Ti provide good adhesion between $\mathrm{Au}$ and $\mathrm{GaN}$. Four Schottky contacts were made on a single piece of GaN. $I-V, C-V$, and DLTS measurements were performed using a Keithley 237 source measurement unit, an Agilent 4285A LCR meter, and a Semilab DLS-83D spectrometer based on lock-in principle, respectively. ${ }^{27}$

\section{RESULT AND DISCUSSION}

\section{A. $\boldsymbol{I}-\boldsymbol{V}$ and $\boldsymbol{C}-\boldsymbol{V}$ Measurements}

Figure 1 displays the representative $A^{2} / C^{2}-V$ data of an $n$-GaN device measured at $75 \mathrm{kHz}$ while the spatial distribution of background concentration of the device is shown in the inset of the same figure. A linear relationship between the

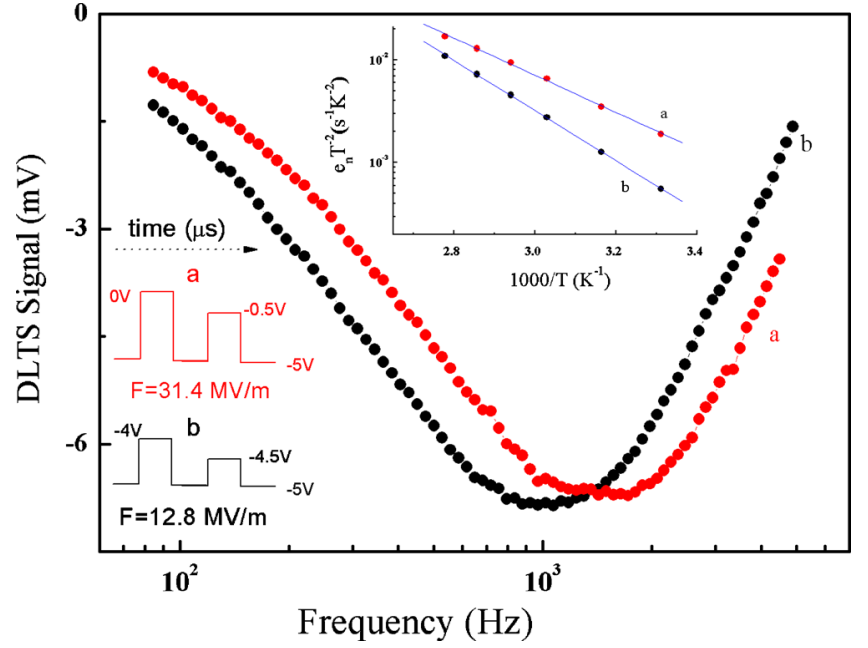

FIG. 2. (Color online) Representative frequency-DLTS spectra displaying the field enhanced emission signatures of an electron trap $E$. The applied excitation pulse conditions for DLTS scans (a), (b) are sketched in the respective colors of the curves, here pulse width, $t_{p}=20 \mu$ s. Inset demonstrates the associated Arrhenius plots at two representative fields.

inversely squared capacitance $\left(A^{2} / C^{2}\right)$ and applied bias $(V)$ clearly supports Schottky behavior of the metalsemiconductor (MS) contacts. The depth profile of the free carrier concentration in the explored area is uniform as follows from the inset of the Fig. 1. From the $A^{2} / C^{2}-V$ data, the built-in potential $\left(V_{b i}\right)$ and free carrier concentration $\left(N_{D}\right)$ of the device were extracted from the $x$-intercept and slope using linear fitting of the data, respectively. ${ }^{28}$ The barrier height was estimated by the equation $\phi_{B}=V_{b i}+V_{o}$, here $V_{o}$ is the potential difference between the Fermi level and the flat tail of the conduction band, given by: $V_{o}=(k T / q) \ln \left(N_{c} / N_{D}\right)$, $N_{C}=2\left(m^{*} k_{B} T / 2 \pi \hbar^{2}\right)^{3 / 2}=3.5 \times 10^{18} \mathrm{~cm}^{-3}$, is the conduction band density of states at room temperature. Consequently, $\phi_{B}$ and $N_{D}$ from the $C$ - $V$ data were found to be $1.08 \mathrm{eV}$ and $1.7 \times 10^{17} \mathrm{~cm}^{-3}$, respectively. The barrier height and ideality factor $n$ were also calculated from the $I-V$ data (not shown here) and the values were $0.53 \mathrm{eV}$ and 4.88, respectively. Because of the different measuring mechanism of the two techniques: $I-V$ and $C-V$ (transport and static, respectively), there is often a discrepancy in the values of $\phi_{B}{ }^{29}$ Additionally, the higher value of $n$ can originate from contact inhomogeneities, which generate interface states to provide multiple paths to the carriers. ${ }^{29,30}$

\section{B. DLTS measurements}

In literature, we can find different experimental methods being used to study the electric field dependence of deep level emission rates. ${ }^{20,31-34}$ Accordingly, we adopted the method described by Makram-Ebeid, ${ }^{34}$ details of it can be found in the DLS-83 User's Manual. ${ }^{35}$ Figure 2 displays the representative frequency scans (curves a and b) obtained from a $n$-GaN sample at two extreme applied fields (12.8 $\mathrm{MV} / \mathrm{m}$ and $31.4 \mathrm{MV} / \mathrm{m}$ ), respectively, while rest of the measuring conditions were maintained as same: $T=360 \mathrm{~K}$, pulse width, $t_{p}=20 \mu \mathrm{s}, U_{R}=-5 \mathrm{~V}, U_{1}=-4 \mathrm{~V}$ and $0 \mathrm{~V}, U_{2}=$ $-4.5 \mathrm{~V}$ and $-0.5 \mathrm{~V}$, respectively. It has to be mentioned here that, due to the high value of the device leakage current at 


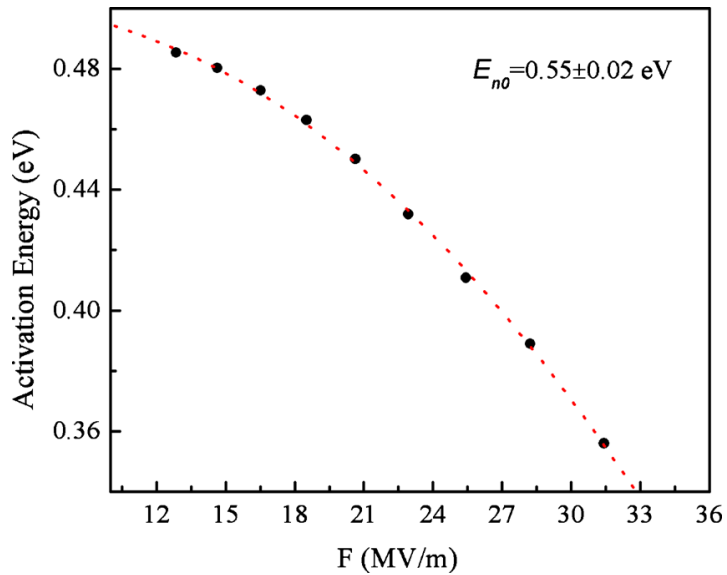

FIG. 3. (Color online) Evidence of pure thermal energy $\left(E_{n 0}\right)$ of the trap $E$ by extrapolating the field dependent data upto zero field value.

applied reverse bias $>-5 \mathrm{~V}$, electric fields in excess of $>31.4 \mathrm{MV} / \mathrm{m}$ could not be applied. An electron level $E$ appears to exhibit qualitative field dependent emission rates $\left(e_{n}\right)$ : the trap peak position shifts with increasing applied field towards higher frequencies with $\Delta \mathrm{f} \sim 550 \mathrm{~Hz}\left(\Delta e_{n}\right.$ $=1190 \mathrm{~s}^{-1}$ ). To have a quantitative look of the observation, we performed several frequency scans at sample temperatures $(300,320,340,350$, and $360 \mathrm{~K})$ with varying applied field. The activation energy of the trap $E$ at a constant field was estimated using an Arrhenius plot. ${ }^{23}$ The representative Arrhenius data associated with the level $E$ at two extreme fields are depicted in the inset of Fig. 2; the field assisted thermal activation energy of the electron trap is found to be 0.35 and $0.48 \mathrm{eV}$ with respect to the applied field of 31.4 and 12.8 MV/m, respectively. The as-calculated activation energies of the trap were plotted versus the applied field yielding the zero field. Consequently zero field energy, $E_{n 0}$ (pure thermal activation energy) to be $0.55 \pm 0.02 \mathrm{eV}$ by extrapolating the data along the field as shown in Fig. 3. This value interestingly happens to be nearly the same activation energy as reported for the electron level $E$ in $n$-GaN showing field dependent emission properties. ${ }^{14-16}$ The reduction in thermal emission energy of the carriers from the trap as a result of applied field is explained by three mechanisms: (1) Poole-Frenkel, ${ }^{23}$ (2) phonon assisted tunneling, ${ }^{36,37}$ and (3) pure tunneling. ${ }^{38}$ Mechanisms (1) and (2) are effective over the field range $10^{6}-10^{8} \mathrm{~V} / \mathrm{m}$ and (3) is significant at high fields $\geq 10^{9} \mathrm{~V} / \mathrm{m}$. Therefore, in present study, we will focus on mechanisms (1) and (2) to explain our results. Qualitatively, Poole-Frenkel theory states that the electron band diagram is slanted and the potential barrier (trap) depth decreases under the applied field as shown in Fig. 4, the emission energy (electron) is henceforth reduced by an amount $\delta E$. However, if the electron has coupling with suitable phonon(s) the emission energy will be even lower and the electron will tunnel through the barrier (phonon-assisted theory). Quantitatively, the effective emission energy of the carriers from the trap depends upon the type and shape of the barrier: relying on the nature of the defect level, Martin et $a l^{38}$ and Vincent et $a l .{ }^{32}$ proposed a Coulomb potential, a square well potential or polarization potential models to fit

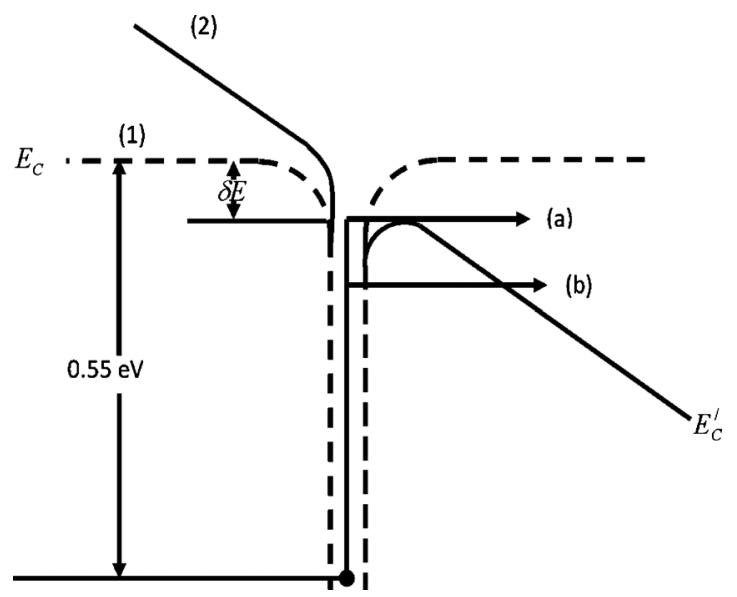

FIG. 4. Electron energy diagram in equilibrium (1) and in the presence of an electric field (2) showing field enhanced electron emission: (a) PooleFrenkel emission and (b) phonon-assisted tunneling.

Poole-Frenkel effect on the emission rates of the carriers. On the other hand, Makram-Ebeid and Lannoo ${ }^{36}$ and Korol, ${ }^{39}$ using time dependent perturbation quantum theory and Wentzel-Kramers-Brillouin (WKB) approximation, respectively, added the contribution of phonons in the square well potential calculations and succeeded to justify the phononassisted tunneling mechanism in emission energy of the carriers from the trap. The mathematical expressions representing the aforesaid potentials exhibiting Poole-Frenkel effect, are listed below: Eqs. (1)-(3) describe the emission rates calculated by three-dimensional Coulombic potential [Eq. (1)], square well potential [Eq. (2)], and polarization potential [Eq. (3)]:

$$
\begin{aligned}
\frac{e_{n}(F)}{e_{n}(0)}= & \frac{1}{\gamma^{2}}\left[e^{\gamma}(\gamma-1)+1\right]+\frac{1}{2} \\
& \text { where } \gamma=\left(q F / \pi \varepsilon_{r} \varepsilon_{o}\right)^{1 / 2} q / k T,
\end{aligned}
$$

$$
\begin{aligned}
\frac{e_{n}(F)}{e_{n}(0)} & =\frac{1}{2 \gamma}\left(e^{\gamma}-1\right)+\frac{1}{2} \quad \text { where } \gamma=q F r / k T, \\
\frac{e_{n}(F)}{e_{n}(0)} & =\exp (\gamma) \text { here } \gamma \\
& =1.649 A^{1 / 5}(q F)^{4 / 5} / k_{B} T \text { and } A=q^{2} \alpha / 8 \pi \varepsilon_{o} \varepsilon_{r}^{2},
\end{aligned}
$$

$$
A=5.12 \times 10^{-32} \mathrm{eV} \mathrm{cm}^{4} .
$$

The emission rates due to phonon-assisted tunneling derived by $\mathrm{Korol}^{39}$ [Eq. (4)] and Makram-Ebeid and Lannoo [Eq. $(5)]^{36}$ are given by

$$
\begin{gathered}
e_{n}=e_{n}^{\infty} \exp \left(-\frac{E_{i}-E}{k T}\right) \exp \left(-\frac{4\left(2 m^{*}\right)^{1 / 2} E^{3 / 2}}{3 q \hbar F}\right) \\
\text { provided: } \frac{q \hbar F}{2\left(2 m^{*}\right)^{1 / 2}}>k T E_{I}^{1 / 2} .
\end{gathered}
$$

The emission rate for quantum model for phonon-assisted tunneling is 


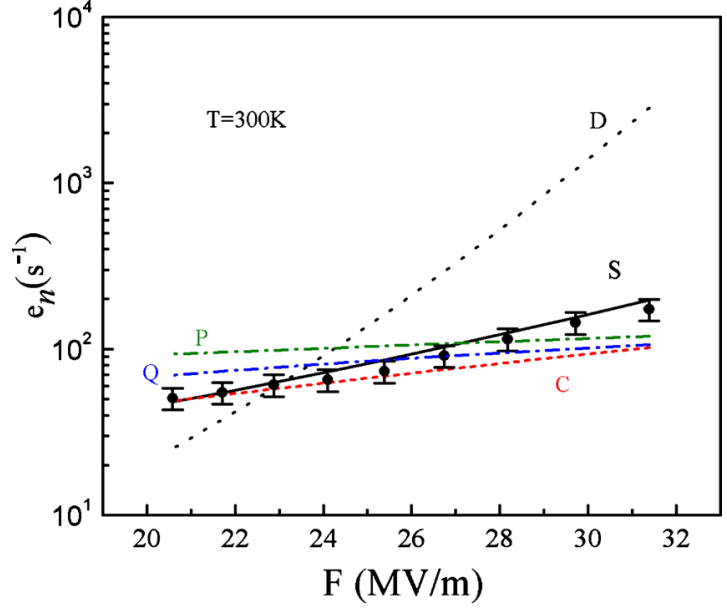

FIG. 5. (Color online) Experimental data (filled squares) is evidenced to fit with square well potential (s), however, other theoretical models [polarization potential (p) and Dirac well potential (d), Coulombic potential (c), semiclassical quantum mechanical (q), deviate from the experimental measurements.

$$
e_{n}=e_{n 0}+\gamma \frac{e^{-A}}{A} \frac{\Delta_{1}}{2}
$$

where $e_{n o}$ is the zero emission rate and $R$ is the tunneling rate, $\Delta_{1}=6.5$,

$$
A=\frac{4 / 3\left(2 m^{*} / \hbar^{2}\right)^{1 / 2}\left(\Delta_{f}\right)^{3 / 2}}{F}=\frac{0.95974}{F},
$$

where $\Delta_{f}=(1 / 4) E_{g}$ and $\gamma$ is an adjustable pre-exponential factor. Here all the constants bear the usual meanings. Using relations (1)-(5), the emission rates were calculated and plotted in Fig. 5 together with the experimental emission data for the observed trap $E$ at sample temperature $360 \mathrm{~K}$. The result reveals that the experimental data are in good agreement with the Poole-Frenkel model associated with the square well potential. Accordingly, the square well fitting was applied on the emission rates carried out at temperatures of 300-360 K, and the results were found to be well consistent. Considering the correlation of the trap $E$ with the square well potential exhibiting Poole-Frenkel effect, the level $E$ has to be identified as a charged impurity. In this perception, we argue that the field dependent emission properties of the electron defect with an activation energy $E_{c}-0.53$ to $E_{c}$ $-0.61 \mathrm{eV}$ reported by Soh et al.,${ }^{14} \mathrm{Wu}$ et al. ${ }^{15}$ and Asghar et al. ${ }^{16}$ might be associated with the charged impurity. The probable candidates could be gallium and/or nitrogen linked with interstitials, antisites, or vacancy complexes. ${ }^{11-21}$ However, the final identification of the impurity is still not clear.

\section{CONCLUSION}

In this study, free standing GaN layers were grown by HVPE and characterized. The metal/semiconductor interface states and field dependent emission signatures of an electron trap $E$ by DLTS have been reported. The field dependent emission rate of electron trap has been reported for the first time, to the best of our knowledge. Several DLTS frequency scans were performed at constant electric field (12.8-31.4 $\mathrm{MV} / \mathrm{m}$ ) and the Arrhenius plots of the data yielded the acti- vation energies of the electron trap in the range of $\left(E_{c}\right.$ $\left.-0.48 \pm 0.02-E_{c}-0.35 \pm 0.02 \mathrm{eV}\right)$, respectively, whereas the extrapolated zero field activation energy of the defect was found to be $0.55 \pm 0.02 \mathrm{eV}$. A number of potential models have been tried to identify the emission mechanism of trap $E$. Poole-Frenkel effect associated with square well potential model was found consistent. Consequently level $E$ was attributed to a charged impurity, and the probable candidates could be gallium and/or nitrogen linked with interstitials, antisites, or vacancy complexes.

\section{ACKNOWLEDGMENTS}

H. Ashraf would like to acknowledge P. Mulder for assisting in diodes fabrication. The research work was funded by Higher Education Commission of Pakistan under Project No. 1019/R\&D/2007 (M. Asghar) and Dutch technology foundation (STW) (Project No. NAF. 06937). Last but not the least, we are thankful to Ms. Elisa Hurwitz of UNC Charlotte, USA for critical reading of the manuscript.

${ }^{1}$ V. A. Dmitriev, K. G. Irvine, and C. H. Carter, Appl. Phys. Lett. 68, 229 (1996).

${ }^{2}$ T. Matsuoka, A. Ohki, T. Ohno, Y. Kawaguchi, J. Cryst. Growth 138, 727 (1994).

${ }^{3}$ S. Nakamura, M. Senoh, S. Nagahama, N. Iwasa, T. Yamada, T. Matsushita H. Kiyoku, and Y. Sugimoto, Jpn. J. Appl. Phys., Part 2 35, L74 (1996).

${ }^{4}$ M. A. Reshchikov and H. Morkoç, J. Appl. Phys. 97, 061301 (2005).

${ }^{5}$ T. Miyajima, H. Yoshida, K. Yanashima, T. Yamaguchi, T. Asatsuma, K. Funato, S. Hashimoto, H. Nakajima, M. Ozawa, T. Kobayashi, S. Tomiya, T. Asano, S. Uchida, S. Kijima, T. Tojyo, T. Hino, and M. Ikeda, Mater. Sci. Eng., B 82, 248 (2001).

${ }^{6}$ Y.-F. Wu, A. Saxler, M. Moore, R. P. Smith, S. Sheppard, P. M. Chavarkar, T. Wisleder, U. K. Mishra, and P. Parikh, IEEE Electron Device Lett. 25, 117 (2004).

${ }^{7}$ H. Morkoç, A. di Carlo, and R. Cinjolani, Solid-State Electron. 46, 157 (2002).

${ }^{8}$ C. D. Wang, L. S. Yu, S. S. Lau, E. T. Yu, W. Kim, A. E. Botchkarev, and H. Morko, Appl. Phys. Lett. 72, 1211 (1998).

${ }^{9}$ P. Hacke, P. Ramvall, S. Tanaka, Y. Aoyagi, A. Karumata, K. Horino, and H. Munekata, Appl. Phys. Lett. 74, 543 (1999).

${ }^{10}$ H. M. Chung, W. C. Chung, Y. C. Pan, C. C. Tsai, M. C. Lee, W. H. Chen, W. K. Chen, C. I. Chiang, C. H. Lin, and H. Chang, Appl. Phys. Lett. 76, 897 (2000)

${ }^{11}$ P. Muret, A. Philippe, E. Monroy, E. Munõz, B. Beaumont, F. Omnès, and P. Gibart, Mater. Sci. Eng., B 82, 91 (2001).

${ }^{12}$ P. Muret, C. Ulzhofer, J. Pernot, Y. Cordier, F. Semond, C. Gacquiere, and D. Theron, Superlattices Microstruct. 36, 435 (2004).

${ }^{13}$ Z. Q. Fang, D. C. Look, J. Jasinski, M. Benamara, Z. Lilintal-Weber, and R. J. Molnar, Appl. Phys. Lett. 78, 332 (2001).

${ }^{14}$ C. B. Soh, Z. Chi, A. Ramam, F. Lim, and S. J. Chua, Mater. Sci. Semicond. Process. 4, 595 (2001).

${ }^{15}$ L. Wu, W. E. Meyer, F. D. Auret, and M. Hayes, Physica B 340-342, 475 (2003).

${ }^{16}$ M. Asghar, P. Muret, B. Beaumont, and P. Gibart, Mater. Sci. Eng., B 113, 248 (2004)

${ }^{17}$ D. C. Look, Z. Q. Fang, and B. Claflin, J. Cryst. Growth 281, 143 (2005).

${ }^{18}$ A. Armstrong, A. R. Arehart, D. Green, U. K. Mishra, J. S. Speck, and S. A. Ringel, J. Appl. Phys. 98, 053704 (2005).

${ }^{19}$ M. A. Py, C. Zellweger, V. Wagner, J.-F. Carlin, H.-J. Buehlmann, and M. Ilegms, Phys. Status Solidi A 202, 572 (2005).

${ }^{20}$ D. V. Lang, J. Appl. Phys. 45, 3023 (1974).

${ }^{21}$ J. Pernot and P. Muret, J. Appl. Phys. 103, 023704 (2008).

${ }^{22}$ D. Pons, J. Appl. Phys. 55, 3644 (1984).

${ }^{23} \mathrm{P}$. Blood and J. W. Orton, The Electrical Characterization of Semiconductors: Majority Carriers and Electron States (Academic, London, 1992), Chap. 8.

${ }^{24}$ A. Y. Polyakov, N. B. Smirnov, A. V. Govorkov, A. V. Markov, Q. Sun, Y. Zhang, C. D. Yerino, T.-S. Ko, I.-H. Lee, and J. Han, Mater. Sci. Eng., B 
166, 220 (2010).

${ }^{25}$ H. Ashraf, J. L. Weyher, G. W. G. van Dreumel, A. Gzregorzych, and P. R. Hageman, J. Cryst. Growth 310, 3957 (2008).

${ }^{26}$ H. Ashraf, R. Kudrawiec, J. L. Weyher, J. Serafinczuk, J. Misiewicz, and P. R. Hageman, J. Cryst. Growth 312, 2398 (2010).

${ }^{27}$ G. Ferenczi and J. Kiss, Acta Physiol. Acad. Sci. Hung. 50, 285 (1981).

${ }^{28}$ D. K. Schroder, Semiconductor Material and Device Characterization, 3rd ed. (Wiley, New York, 2006), Chap. 4.

${ }^{29}$ J. Werner, K. Ploog, and H. J. Queisser, Phys. Rev. Lett. 57, 1080 (1986).

${ }^{30}$ P. Muret, D. Elguennouni, M. Missous, and E. H. Rhoderick, Appl. Phys. Lett. 58, 155 (1991).

${ }^{31}$ J. M. Herman III and C. T. Sah, Phys. Status Solidi A 14, 405 (1972).
${ }^{32}$ G. Vincent, A. Chantre, and D. Bois, J. Appl. Phys. 50, 5484 (1979).

${ }^{33}$ H. Lefèvre and M. Schulz, J. Appl. Phys. 12, 45 (1977).

${ }^{34}$ S. Makram-Ebeid, Appl. Phys. Lett. 37, 464 (1980).

${ }^{35}$ DLS-83D User's Manual (Semilab Rt. Budapest, Hunagry,) vol. 1. appendix $1 / 9$.

${ }^{36}$ S. Makram-Ebeid and M. Lannoo, Phys. Rev. Lett. 48, 1281 (1982).

${ }^{37}$ S. D. Ganichev, E. Ziemann, and W. Prettl, Phys. Rev. B 61, 10361 (2000).

${ }^{38}$ P. A. Martin, B. G. Streetman, and K. Hess, J. Appl. Phys. 52, 7409 (1981).

${ }^{39}$ E. N. Korol, Sov. Phys. Solid State 19, 1327 (1977); see Ref. 5 in Ref. 32 also. 